\title{
ASSESSING SYSTEMS OF SYSTEMS' PERFORMANCE USING A HIERARCHICAL EVALUATION PROCESS
}

\author{
Rahim Jassemi-Zargani* \\ Defence Research \& Development Canada (DRDC) Ottawa \\ Ottawa, ON, Canada \\ E-mail: Rahim.Jassemi@drdc-rddc.gc.ca \\ Nathan Kashyap \\ Defence Research \& Development Canada (DRDC) Ottawa \\ Ottawa, ON, Canada \\ E-mail: Nathan.Kashyap@drdc-rddc.gc.ca
}

\begin{abstract}
As the course and direction of military conflicts continue to change at the strategic, operational and tactical levels, decision makers need to assess their capability more rapidly, with higher levels of trust, fidelity and effectiveness. Decision makers receive a large number of proposals or acquisition requests for new or upgrading systems, processing and related infrastructure improvements. They review, evaluate and assess the relative and absolute effectiveness of each proposal and mitigate any risk factors associated with each potential acquisition and the integration of additional capabilities to the overall system. The capabilities can be broken down into performance, integration and interoperability of system of systems in order to achieve ultimate performance to address their mission requirements. This paper will present the design of a decision support tool called the Virtual Intelligence, Surveillance and Reconnaissance (ISR) Evaluation Environment (VIEE) and the methodology that is used for the overall performance evaluation of different ISR system of systems based on Analytical Hierarchy Process (AHP) and Analytical Network Process (ANP) methods. A scenario will be used to describe how the tools function in VIEE while demonstrating the effectiveness of system of systems performance.
\end{abstract}

Keywords: Defence, Assessment, Command \& Control, Exploitation, AHP, ISR

\section{Introduction}

Overall performance evaluation of integrated systems of systems is a complex problem. A potential solution is to apply a hierarchical process in order to address all criteria levels related to mission performance. The difficulty of scoping such an evaluation exists in the underlying details, as not only the systems themselves are subject to evaluation, but also the process, exploitation, dissemination and infrastructure that is part of each system of systems needs to be accounted for. The scope of the assessment needs to be manageable yet able to maintain a level of reliability and fidelity to provide meaningful results. The level of complexity is not only limited to identifying the different layers of evaluation criteria, but also to the relationships between the criteria and sub-criteria levels.

A capability, termed the Virtual Intelligence, Surveillance and Reconnaissance (ISR) Evaluation Environment (VIEE), has been developed, which allows users to model systems, build the scenarios used for evaluation, apply exploitation tools to process the systems data, and assessment tools (build figure of merits and influence diagrams) to evaluate the overall system of systems mission performance and

\footnotetext{
${ }^{*}$ Corresponding author
} 
effectiveness. As part of this environment, there are two methods that are considered for the evaluation: one based on Analytical Hierarchy Process (AHP), and the other based on Analytical Network Process (ANP) (Saaty, 1995) (Saaty and Vargas, 2006). Input from Subject Matter Experts (SME) are collected and used to populate the comparison matrices used in the AHP and ANP methods. This allows for differing human perceptions in judging the comparisons between criteria. The AHP method allows the decision makers to aggregate all their criteria and sub-criteria to a higher level for evaluations where there is no interdependence (horizontal) relationship between criteria and sub-criteria levels. On the other hand the ANP method does allow for dependency (horizontal) relationships between criteria and sub-criteria. This paper will present the design of VIEE and the methodology used for the overall performance evaluation of different ISR system of systems, and is structured in the following sections: VIEE design, Performance evaluation method, scenario, modeling $\&$ analysis, and conclusions.

\section{VIEE design}

VIEE is an environment which provides a capability to evaluate the performance of ISR system of systems. The design is based on enabling users of VIEE to evaluate sensor performance in a locationindependent manner. In order to accomplish this, the underlying architecture is based on a Service Oriented Architecture (SOA). All web services supported by VIEE share a common database and provide access to VIEE data and exploitation tools. To limit the use of resources on a single machine, the technique of load balancing can be applied to distribute the services and tools across a number of workstations enabling faster processing of evaluation results (Jassemi et al, 2010). VIEE consists of three main components: VIEE client, web services, and modelling and simulation.

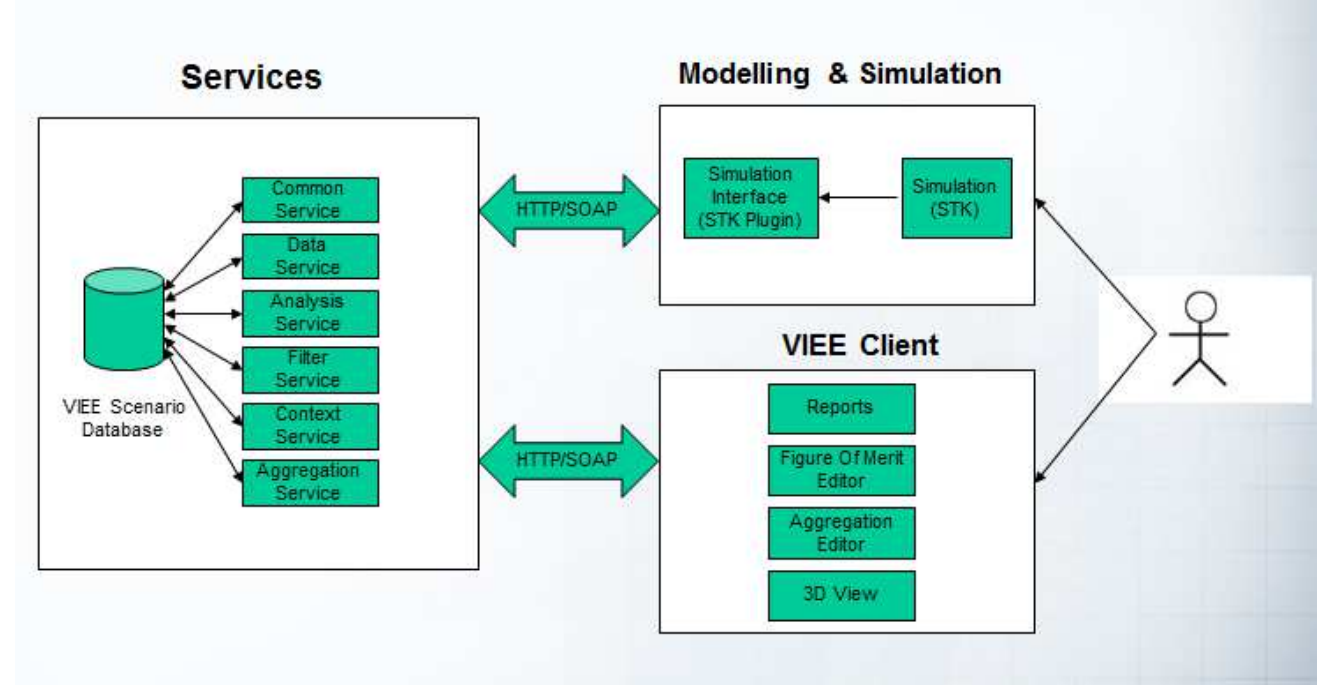

Figure 1. High level Design of VIEE

\subsection{VIEE client}

The client is a graphical user interface that allows the user to interact with the web services that have access to the scenario data. The client can be installed anywhere on a network where it can access the web services. A client makes a request through the interface and the VIEE web services perform the operation on behalf of the client. A graphical representation of the high-level design of VIEE is shown in Figure 1. The VIEE client allows the user to interact with VIEE environment, to access the tools such as setting the context environment, creating the Figures of Merit (FOM) and influence diagram:

\section{- Context:}




\section{R. Jassemi, N. Kashyap/ Assessing System of Systems}

The VIEE context is an interface in the client where a user can apply data processing and exploitation tools to existing scenarios in the database. This allows a user to customize their scenario for evaluation with varying levels of fidelity. In addition to this, there exists properties for systems that cannot be modeled but rather input as properties for the system-such as cost and availability—-that a user may want to include in the influence diagram for their evaluation.

- Figures of Merit (FOM):

Users of VIEE can interact with the application services through an interface that supports web service access of the data. The evaluation process using AHP or ANP begins by creating figures of merit to evaluate the performance of the system. A figure of merit can be simple or very complex. With the aid of a graphical editor, a user can simply drag and drop components onto the design pane to create figure of merits. Once a FOM has been created, the user can execute it using the report wizard. The report wizard will execute the FOM and generate a graphical representation of the results by producing a quantitative result in a chart. The backend processing of this request is sent to the analysis service which will calculate and return the result to client.

- Influence Diagram:

An influence diagram is used to select and apply the AHP or ANP to evaluate mission performance using criteria and sub-criteria. In this context, sub-criteria are the low level leaf nodes in the influence diagram that refer to figures of merit. Similar to the FOM generation, the user can drag and drop criteria and sub-criteria onto the design pane to build the influence diagram. An aggregation wizard is used to execute the influence diagram and produce results to the client.

\subsection{VIEE services}

The services that the VIEE supports are:

- Common service: Shared library of services that other services can access.

- Data service: Provides a mechanism to move data into, and out of, the VIEE database.

- Analysis Service: Provides tools to perform analysis on the contents of scenarios from the VIEE database. This includes figure of merit creation, retrieval, execution, debugging, and aggregation support.

- Filter Service: Provides a client with the ability to filter scenario data

- Context Service: Provides a client with the ability to customize scenarios for analysis. This includes incorporating trackers, correlation algorithms, and post processing tools.

- Aggregation Service: This service supports building influence diagrams and aggregating all criteria and sub-criteria using AHP and ANP.

\subsection{Modeling and simulation}

Modeling and simulation (M\&S) is used to build ISR models and generate performance reports which are used as input for the evaluation of the ISR systems in VIEE. This is described further in Section 4, by working through a scenario.

\section{Performance evaluation method}

The evaluation of systems of systems' performance is based on a hierarchical method, which takes into account the desired requirement and defines them in lower level Measures of Performance (MOPs) or 
FOMs. These MOPs are aggregated using weights obtained from subject matter experts (SMEs) using Saaty's AHP or ANP methods (Saaty, 1995) (Saaty and Vargas, 2006).

\subsection{FOMs weight factor}

The AHP method provides a clear, traceable means of eliciting subjective assessments and determining a set of overall weights for a multi decision criteria analysis. Individual judgments from the SMEs are collected and, using the AHP, a set of weights is produced for the criteria and sub-criteria. A check is also performed to ensure that the individual SME judgments are consistent to within an acceptable level. Figure 2 shows the process that is required to achieve an acceptable set of weights. If there is a strong inconsistency between individual SME judgments, the process allows the SMEs to re-evaluate their responses, identify the reasons for their inconsistency and make corrections as required (Saaty, 1995) (Jassemi, Bourdon and Fong, 2011).

One of the strongest attributes of the AHP is that it uses a pairwise comparison analysis method to identify the priority value or the weight scale of individual measures. For each SME, several comparison matrices can be created (one at the criteria level and one for each of the groupings at the sub-criteria level). Table 1 shows a generic example of a comparison matrix for four FOMs, where each SME is required to provide values for $\xi_{1}$ to $\xi_{6}$ based on relative importance of each category converted into a corresponding numerical score. After normalization, and passing the consistency test as shown in Figure 2 , the computed weights will be accepted and used for evaluation

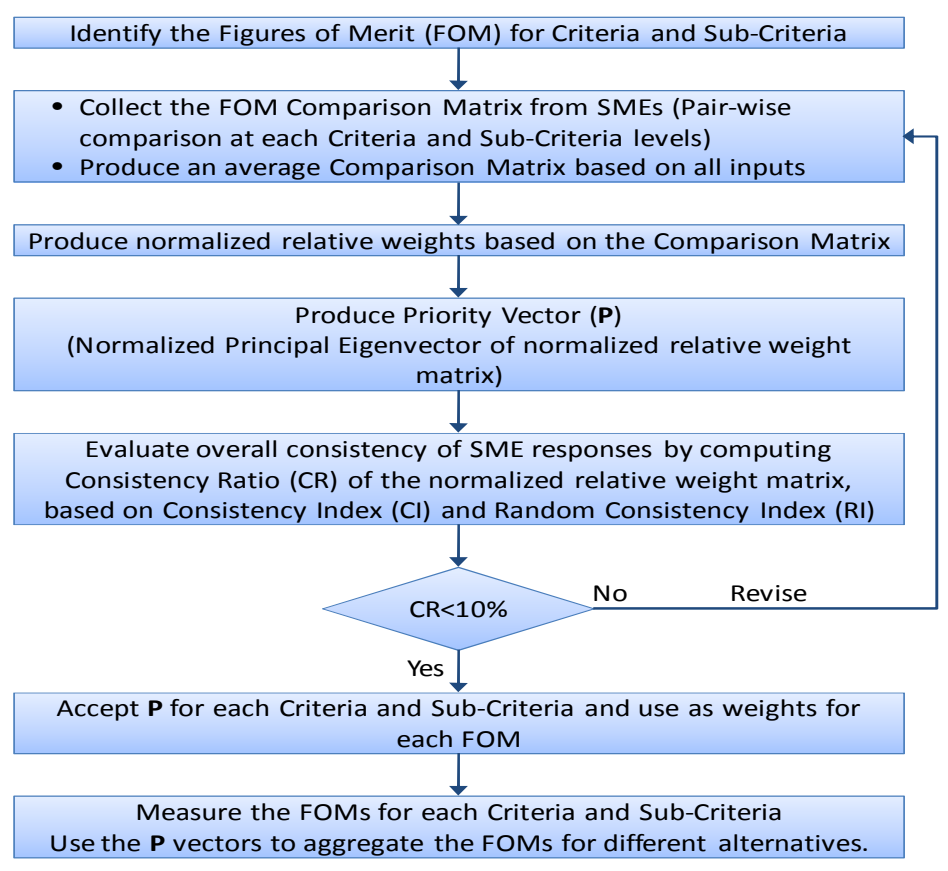

Figure 2. Analytical Hierarchy Process

\subsection{Aggregation process}

The process for aggregating the FOMs into an overall measure of system effectiveness is shown in Figure 3 , where $W_{i}$ is the requirement dependent set of weights obtained using AHP and $S_{\mathrm{i}}$ represents the values of $\mathrm{FOM}_{\mathrm{i}}$ normalized to the interval $(0,1)$ (Jassemi, Bourdon and Fong, 2011). 
Table 1. Generic example of comparison matrix

\begin{tabular}{lcccc}
\hline \hline & $\sum^{F}$ & $\sum^{N}$ & $\sum^{\infty}$ & $\sum^{T}$ \\
& 1 & $\xi_{1}$ & $\xi_{2}$ & $\xi_{3}$ \\
\hline $\mathbf{F O M}_{1}$ & $1 / \xi_{1}$ & 1 & $\xi_{4}$ & $\xi_{5}$ \\
\hline $\mathbf{F O M}_{2}$ & $1 / \xi_{2}$ & $1 / \xi_{4}$ & 1 & $\xi_{6}$ \\
\hline $\mathbf{F O M}_{3}$ & $1 / \xi_{3}$ & $1 / \xi_{5}$ & $1 / \xi_{6}$ & 1 \\
\hline $\mathbf{F O M}_{4}$ & & &
\end{tabular}

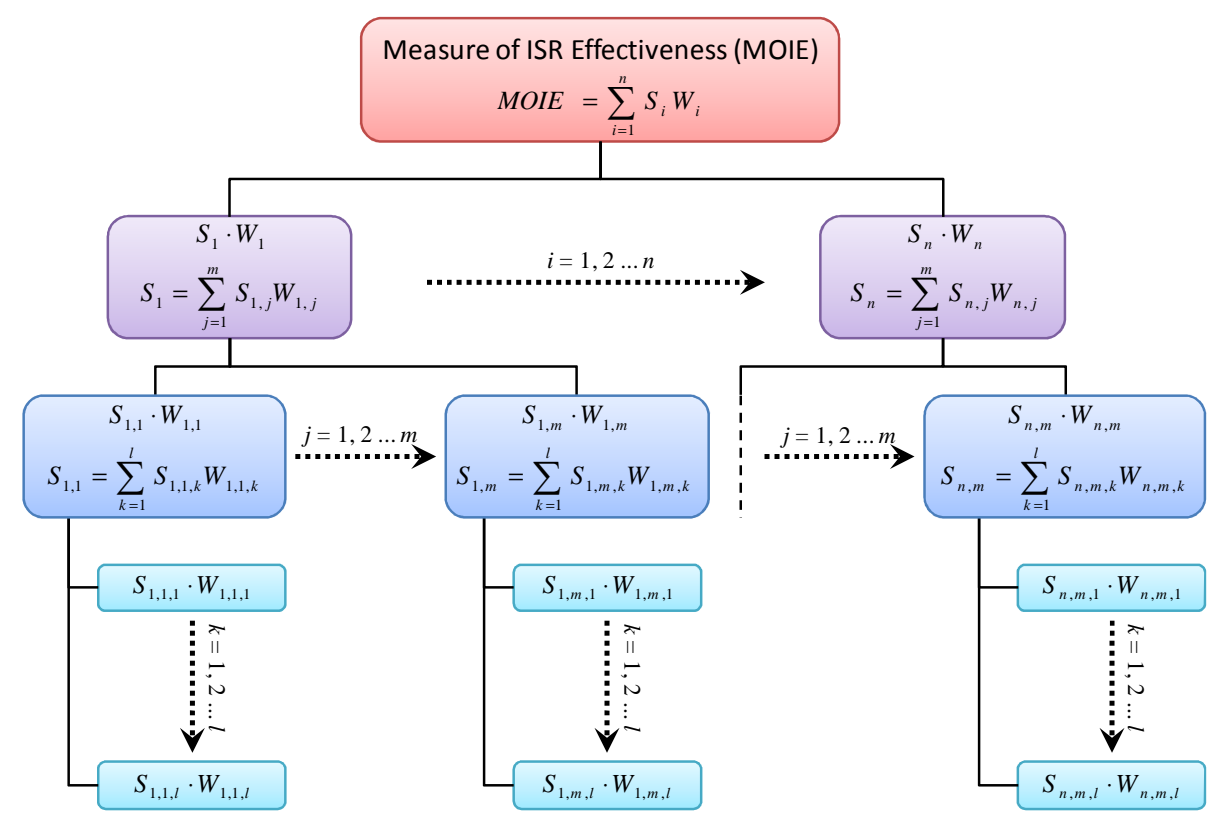

Figure 3. Measure of ISR systems effectiveness computation process based on AHP.

\section{Scenario, modeling and analysis}

To evaluate systems of systems' performance using AHP methodology, a walk-through of the development and evaluation for an ISR scenario is used to demonstrate this concept. Here, an example is used to illustrate how to conduct mission performance evaluation by adding new ISR systems to an existing ISR capability using the evaluation method proposed in this paper.

\subsection{ISR system of systems scenario}

The scenario is built with ISR sensors, platforms, flight plans, routes, and areas of interests. The scenario is based on an Arctic surveillance mission, where a ship travels the Arctic on a pre-determined route.

Various areas of interests were identified. ISR sensor models and platforms were built to produce their mission performance. Prior to building the FOMs and influence diagram, it is helpful to determine the requirements for the scenario as shown in Table 2. Figure 4 is a graphical representation of the scenario, which includes the areas of interest, the systems and the simulation environment. 
Table 2. Scenario requirements

\begin{tabular}{|l|}
\hline \multicolumn{1}{|c|}{ Requirement } \\
\hline Coverage \\
\hline Revisit every 5 hours \\
\hline Coverage percentage $>90 \%$ \\
\hline Total time in area of interest \\
\hline Detection \\
\hline Maximum revisit gap $<10$ hours \\
\hline Average revisit gap $<5$ hours \\
\hline Total number of detections \\
\hline Cost \\
\hline Acquisition cost $<\$ 1$ million \\
\hline Operations cost per year $<\$ 2$ million \\
\hline Maintenance cost $<\$ 100 \mathrm{~K}$ \\
\hline
\end{tabular}

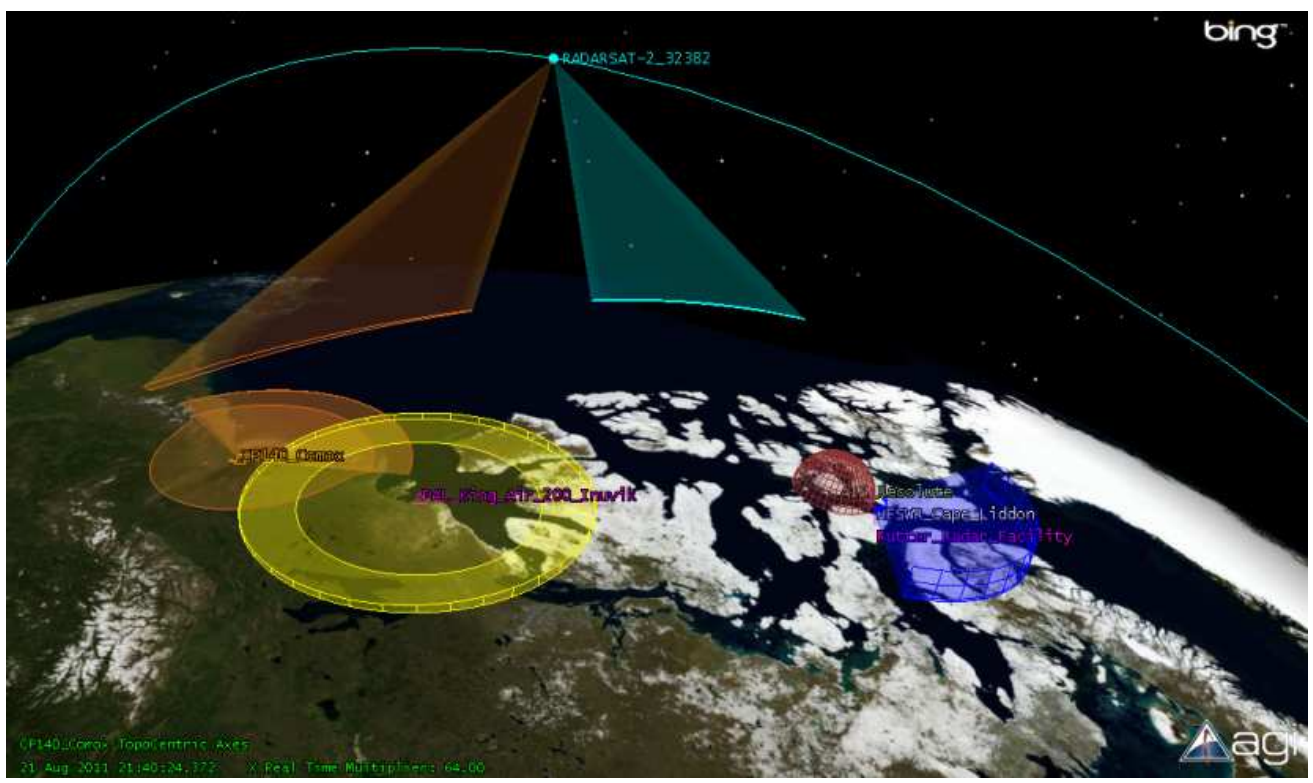

Figure 4. Systems of systems scenario

\subsection{FOMs weight selection}

The FOMs selections are based on operational requirements, which could be represented as criteria. For this scenario, there are three criteria that have been selected: coverage performance, detection performance, and cost. Each criteria has number of related sub-criteria, shown in Table 3. 
Table 3. The Criteria and related sub-criteria

\begin{tabular}{|c|c|c|c|}
\hline & Criteria & & \\
\hline \multirow{4}{*}{ Sub-Criteria } & Coverage & Detection & Cost \\
\hline & Mean Coverage Gap /Revisit & Total number of Detection & Acquisition \\
\hline & Mean Coverage Percentage & Maximum detection Gap & Maintenance \\
\hline & Mean Coverage Time & Mean Detection Gap & Operation \\
\hline
\end{tabular}

Figure 5 shows the comparison matrix input in VIEE, which are used by SMEs to input their recommended weightings. Input data is used to compute the criteria and sub-criteria FOM's weightings.

\begin{tabular}{|c|c|c|c|}
\hline \multicolumn{3}{|c|}{ Comparison Editor: Coverage } & \begin{tabular}{|l|l|l|}
$\square$ & 回 & $x$ \\
\end{tabular} \\
\hline & Mean Coverage Gap & Mean Coverage $\%$ & Mean Coverage Time \\
\hline Mean Coverage Gap & & 2 & 0.5 \\
\hline .8 Mean Coverage $\%$ & & & 3 \\
\hline \multicolumn{4}{|l|}{ Mean Coverage Time } \\
\hline Cancel & & & $\mathrm{OK}$ \\
\hline
\end{tabular}

Figure 5. VIEE comparison matric

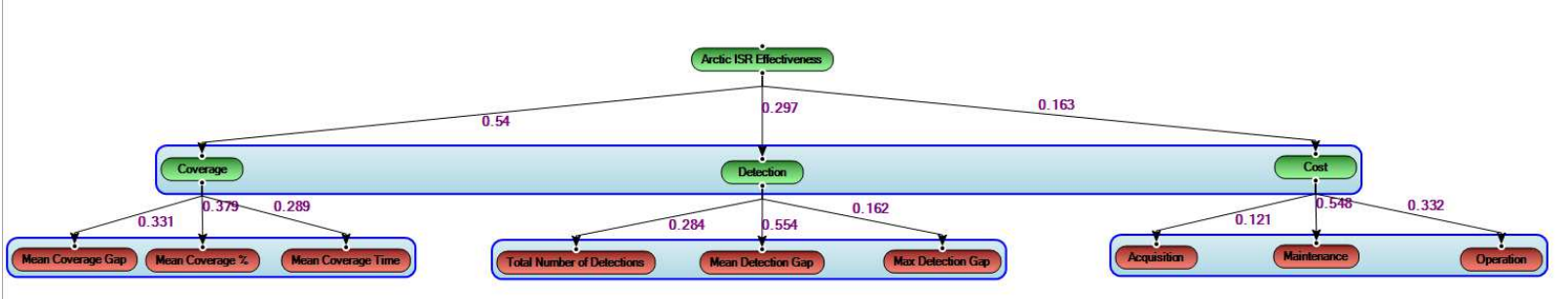

Figure 6. Influence Diagram

\subsection{Modeling influence diagram}

The influence diagram takes into account all criteria and sub-criteria FOMs based on Table 3. A graphical representation of the influence diagram displaying the relationship between the goal, criteria and subcriteria is shown in Figure 6. Also, the weights based on the results of the comparison matrices are shown on the arrows between sub-criteria, criteria and the goal.

\subsection{Results}

Once the influence diagram has been created, the aggregation report wizard is used to generate a graphical representation of the result. The aggregation process calculates every FOM value based on the inputs provided and feeds the results up the chain in the influence diagram. The inputs for the influence diagram consist of creating different alternatives of the system of systems. The alternatives selected in this example from the scenario are listed on the x-axis of Figure 7. Each criteria node takes the results from lower level FOM nodes and calculates the criteria value by using the comparison matrices values. This process continues until the top level node is reached and the final result is determined. The final 
results shown in Figure 7, gives the user a graphical representation of the overall system of systems effectiveness based on the selected criteria and sub-criteria.

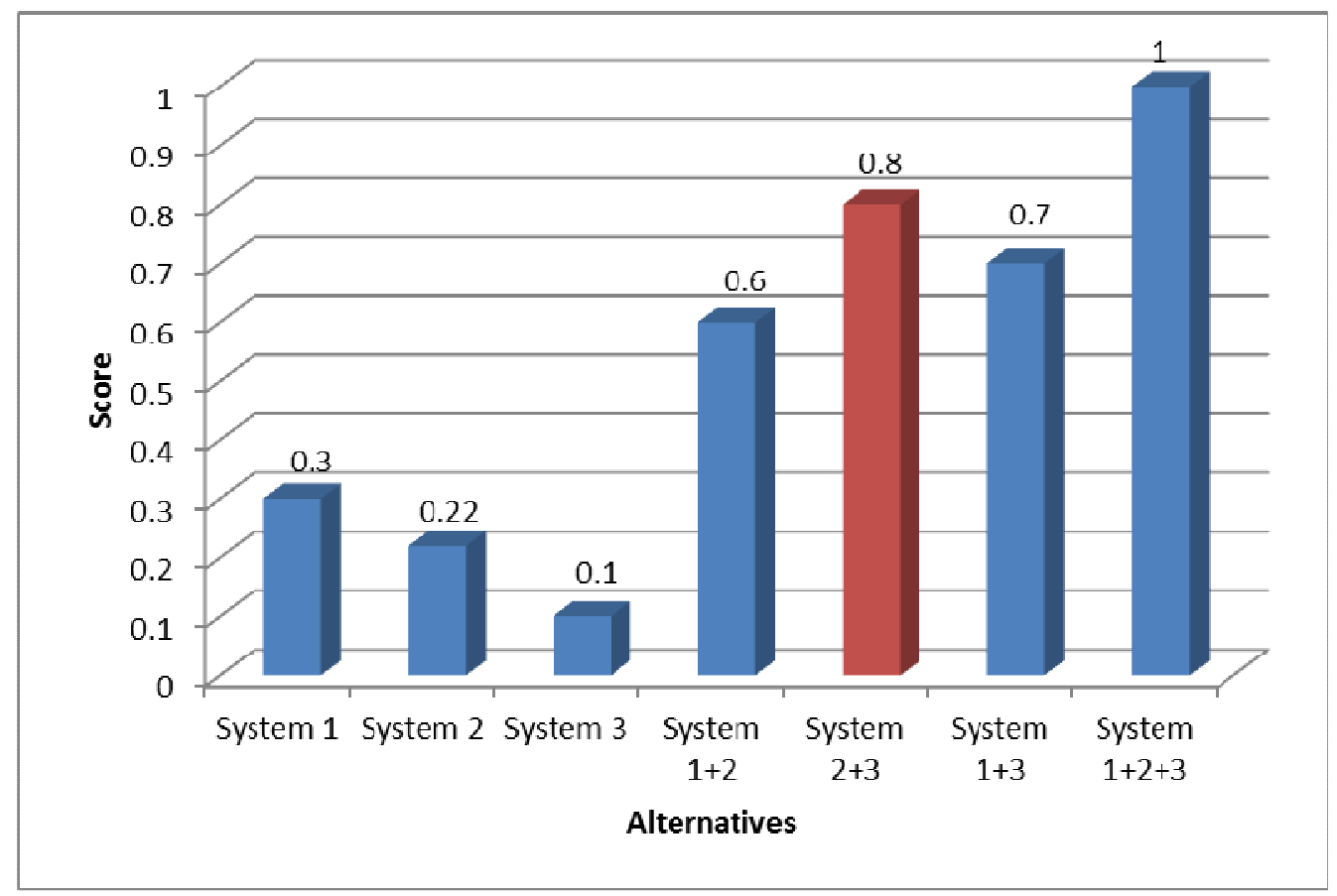

Figure 7. Overall ISR System Alternative Effectiveness

\subsection{Analysis}

One method of determining the "best" alternative is to apply the scenario requirements to the list of alternatives. Table 4 displays scenario requirements on the left with the different system of systems alternatives on the right. The checkmark shows that the requirement is met by the alternative. "N/A" means that the alternative does not consider an upper or lower bound to measure the criteria against. In this scenario, alternative "System $2+3$ " and "System $1+3$ " would be considered because they meet all scenario requirements. Given this, the most effective system of systems alternative would then be "System 2+3" since it has a higher computed aggregation score when considering the AHP model.

\section{Conclusions and recommendations}

The development of this environment with the analysis toolset for performance evaluation of system of systems will enable decision makers to access the information they require to make informed decisions. The environment provides a traceable, repeatable and customizable capability to adopt mission requirements, and conduct analysis on the mission performance of any system of systems alternatives. The AHP method has been fully implemented. The ANP method has not been implemented but is planned for future development in this environment. 
Table 4. Alternative ISR systems that meet scenario requirements

\begin{tabular}{|c|c|c|c|c|c|c|c|}
\hline Requirement & & & & Iternativ & & & \\
\hline Coverage & $\begin{array}{c}\text { System } \\
1\end{array}$ & $\begin{array}{c}\text { System } \\
2\end{array}$ & $\begin{array}{c}\text { System } \\
3\end{array}$ & $\begin{array}{l}\text { System } \\
1+2\end{array}$ & $\begin{array}{c}\text { System } \\
2+3\end{array}$ & $\begin{array}{c}\text { System } \\
1+3\end{array}$ & $\begin{array}{l}\text { System } \\
1+2+3\end{array}$ \\
\hline $\begin{array}{l}\text { AOI revisit every } 5 \\
\text { hours }\end{array}$ & $\checkmark$ & & & $\checkmark$ & $\checkmark$ & $\checkmark$ & $\checkmark$ \\
\hline $\begin{array}{l}\% \text { AOI coverage > } \\
90 \%\end{array}$ & $\checkmark$ & $\checkmark$ & & $\checkmark$ & $\checkmark$ & $\checkmark$ & $\checkmark$ \\
\hline $\begin{array}{l}\text { Total time AOI } \\
\text { covered }\end{array}$ & N/A & N/A & N/A & N/A & N/A & N/A & N/A \\
\hline Detection & & & & & & & \\
\hline $\begin{array}{l}\text { Maximum revisit gap } \\
<10 \text { hours }\end{array}$ & & & $\checkmark$ & & $\checkmark$ & $\checkmark$ & $\checkmark$ \\
\hline $\begin{array}{l}\text { Average revisit gap }< \\
5 \text { hours }\end{array}$ & $\checkmark$ & & & & $\checkmark$ & $\checkmark$ & $\checkmark$ \\
\hline $\begin{array}{l}\text { Total number of } \\
\text { detections }\end{array}$ & N/A & N/A & N/A & N/A & N/A & N/A & N/A \\
\hline Cost & & & & & & & \\
\hline Acquisition & $\checkmark$ & $\checkmark$ & $\checkmark$ & & $\checkmark$ & $\checkmark$ & \\
\hline $\begin{array}{l}\text { Operations cost per } \\
\text { year }<\$ 2 \text { million }\end{array}$ & $\checkmark$ & $\checkmark$ & $\checkmark$ & & $\checkmark$ & $\checkmark$ & \\
\hline $\begin{array}{l}\text { Maintenance cost < } \\
\$ 100 \mathrm{~K}\end{array}$ & $\checkmark$ & $\checkmark$ & $\checkmark$ & & $\checkmark$ & $\checkmark$ & \\
\hline
\end{tabular}

\section{REFERENCES}

Jassemi-Zargani, R., Bourdon, S., \& Fong, V. (2011), A Hierarchical Evaluation of Space-Based Systems Performance, DRDC Ottawa, SL 2011-092, Proceedings of 2nd International Conference Space Technology (ICST).

Jassemi-Zargani, R, Robbins, W., Helleur, C., Bourdon, S., Kashyap, \& N., Campbell, D. (2010), Virtual Intelligence, Surveillance and Reconnaissance Evaluation Environment, SCSC Conf., Ottawa, SL2010113.

Saaty, T.L. (1995). Decision Making for Leaders: The Analytic Hierarchy Process for Decisions in a Complex World. RWS Publications, Pittsburgh, PA, USA.

Saaty, T., \& Vargas, L. (2006), Decision making with the Analytic network Process, Springer's International Series Publication. 